\title{
Universally Image Partition Regularity
}

\author{
Dibyendu De \\ School of Mathematics, University of Witwatersrand \\ Private Bag 3, Wits 2050, South Africa \\ dibyendude@gmail.com \\ Ram Krishna Paul \\ Department of Mathematics, Jadavpur University \\ Kolkata-32, India \\ rmkpaul@gmail.com
}

Submitted: Sep 1, 2008; Accepted: Oct 29, 2008; Published: Nov 14, 2008

Mathematics Subject Classifications: Primary 54D35; Secondary 22A15, 05D10, 54D80.

\begin{abstract}
Many of the classical results of Ramsey Theory, for example Schur's Theorem, van der Waerden's Theorem, Finite Sums Theorem, are naturally stated in terms of image partition regularity of matrices. Many characterizations are known of image partition regularity over $\mathbb{N}$ and other subsemigroups of $(\mathbb{R},+)$. In this paper we introduce a new notion which we call universally image partition regular matrices, which are in fact image partition regular over all semigroups and everywhere. We also prove that such matrices exist in abundance.
\end{abstract}

\section{Introduction}

Many of the classical results of Ramsey Theory are naturally stated in terms of image partition regularity of matrices. We start this discussions with the following definition of image partition regularity.

Definition 1.1 Let $S$ be a subsemigroup of $(\mathbb{R},+)$, let $u, v \in \mathbb{N}$, and let $A$ be a $u \times v$ matrix with entries from $\mathbb{Q}$. Then $A$ is image partition regular over $S$ (abbreviated IPR/S) if and only if, whenever $S \backslash\{0\}$ is finitely colored there exists $\vec{x} \in S^{v}$ such that the entries of $A \vec{x}$ are monochromatic.

One of the earliest results of Ramsey Theory is Schur's Theorem [9] which says that whenever the set $\mathbb{N}$ of positive integers is partitioned into finitely many classes (or finitely colored) there exist $x$ and $y$ such that $x, y$, and $x+y$ are contained in one cell of the 
partition (or are monochromatic). Schur's theorem may also be viewed as saying that the matrix

$$
\left(\begin{array}{ll}
1 & 0 \\
0 & 1 \\
1 & 1
\end{array}\right)
$$

is image partition regular over $\mathbb{N}$.

Another of the earliest results of Ramsey Theory is van der Waerden's Theorem [11] which says that whenever $\mathbb{N}$ is finitely colored there must exist arbitrarily long arithmetic progressions. The length five version of van der Waerden's Theorem is clearly equivalent to the statement that the matrix

$$
\left(\begin{array}{ll}
1 & 0 \\
1 & 1 \\
1 & 2 \\
1 & 3 \\
1 & 4
\end{array}\right)
$$

is image partition regular.

Generally image partition regularity of a matrix is considered over certain semigroups. In this paper we are interested in the class of matrices with entries from $\omega$, where $\omega=$ $\mathbb{N} \cup\{0\}$ is the first infinite cardinal, which are image partition regular over all semigroups and everywhere in the sense explained latter. Unless otherwise stated our semigroups will always be considered with the discrete topology.

$[3]$ is a paper concerned with algebraic results in the Stone-Cech compactification of various dense subsemigroups of $(\mathbb{R},+)$ with the discrete topology. In [1] a stronger notion of image partition regularity over various dense subsemigroups of $(\mathbb{R},+)$ has been introduced.

Definition 1.2 Let $S$ be a subsemigroup of $(\mathbb{R},+)$ with $0 \in c \ell(S \backslash\{0\})$, let $u, v \in \mathbb{N}$, and let $A$ be a $u \times v$ matrix with entries from $\mathbb{Q}$. Then $A$ is image partition regular over $S$ near zero if and only if, whenever $S \backslash\{0\}$ is finitely colored and $\delta>0$, there exists $\vec{x} \in S^{v}$ such that the entries of $A \vec{x}$ are monochromatic and lie in the interval $(-\delta, \delta)$.

Being motivated by the definition of image partition regularity near zero we introduce the following definition.

Definition 1.3 Let $(S,+)$ be a semigroup and $\mathcal{A} \subseteq \mathcal{P}(S)$ satisfying the following properties:

(1) $(\forall A \in \mathcal{A})(\forall B \in \mathcal{A})(A \cap B \in \mathcal{A})$;

(2) $\mathcal{A} \neq \emptyset$ and $\emptyset \notin \mathcal{A}$;

(3) $(\forall A \in \mathcal{A})(\forall a \in A)(\exists B \in \mathcal{A})(a+B \subseteq A)$; and

(4) $(\forall A \in \mathcal{A})(\exists B \in \mathcal{A})(B+B \subseteq A)$. 
Let $M$ be a $u \times v$ matrix with entries from $\omega$. Then $M$ is said to be image partition regular over $S$ with respect to $\mathcal{A}$ (abbreviated $I P R / S_{\mathcal{A}}$ ) if whenever $S=\bigcup_{i=1}^{r} C_{i}$ and $A \in \mathcal{A}$ then there exists $\vec{x} \in S^{v}$ and $i \in\{1,2, \cdots, r\}$ such that $M \vec{x} \subseteq C_{i} \cap A$.

For some explanations we mention that in the case of image partition regularity near zero over a dense subsemigroup $S$ of $\mathbb{R}$, one has $\mathcal{A}=\{(-\delta, \delta) \cap S: \delta>0\}$.

From now on by a pair $(S, \mathcal{A})$ we shall always mean a semigroup $S$ with $\mathcal{A} \subseteq \mathcal{P}(S)$ satisfying the above four properties. Further any matrix will be considered with entries from $\omega$.

Definition 1.4 A $u \times v$ matrix $M$ is said to be universally image partition regular if given any pair $(S, \mathcal{A}), M$ is image partition regular over $S$ with respect to $\mathcal{A}$.

In the following discussions we shall observe that for matrices of finite order image partition regularity and universally image partition regularity are the same notion.

Lemma 1.5 Let $(S, \mathcal{A})$ be a pair and $M$ be a $u \times v$ matrix with entries from $\omega$. Then $M$ is image partition regular over $\mathbb{N}$ implies that $M$ is $I P R / S_{\mathcal{A}}$.

Proof. Let $S=\bigcup_{i=1}^{r} C_{i}$ and $A \in \mathcal{A}$. By a standard compactness argument (see [5, Section 5.5] ) there exists $k \in \mathbb{N}$ such that whenever $\{1,2, \cdots, k\}=\bigcup_{i=1}^{r} D_{i}$ there exists $\vec{x} \in\{1,2, \cdots, k\}^{v}$ and $i \in\{1,2, \cdots, r\}$ such that $M \vec{x} \in\left(D_{i}\right)^{u}$. Now by (1) and (4) of Definition 1.3 we can choose $B \in \mathcal{A}$ such that $i B \subseteq A$ for all $i \in\{1,2, \cdots, k\}$. In fact we can do this by induction. Let this be true for $n \in \mathbb{N}$, and we choose $C \in \mathcal{A}$ such that $i C \subseteq A$ for all $i \in\{1,2, \cdots, n\}$. Then by (4) of Definition 1.3, for $C \in \mathcal{A}$ we can choose $D \in \mathcal{A}$ such that $D+D \subseteq C$. By (1), $B=C \cap D \in \mathcal{A}$, which does the rest. To this end let us pick $z \in B$. For each $i \in\{1,2, \cdots, r\}$ let us set $D_{i}=\left\{t \in\{1,2, \cdots, k\}: t z \in C_{i}\right\}$. Then $\{1,2, \cdots, k\}=\bigcup_{i=1}^{r} D_{i}$. So there exists $\vec{x} \in\{1,2, \cdots, k\}^{v}$ and $i \in\{1,2, \cdots, r\}$ such that $M \vec{x} \in\left(D_{i}\right)^{u}$. Put $\vec{y}=z \vec{x}$. Then $M \vec{y} \in\left(C_{i} \cap A\right)^{u}$.

As an immediate corollary of the above lemma we get the following.

Corollary 1.6 Let $M$ be $a u \times v$ matrix with entries from $\omega$. Then $M$ is universally image partition regular if and only if it is image partition regular over $\mathbb{N}$.

To end this introductory discussions let us discuss the algebra of the Stone-Čech compactification of a discrete semigroup. If $S$ is a discrete space, we take the points of the Stone-Čech compactification $\beta S$ of $S$ to be the ultrafilters on $S$, identifying the principal ultrafilters with the points of $S$ (and thus pretending that $S \subseteq \beta S$ ). Given a set $A \subseteq S, \bar{A}=\{p \in \beta S: A \in p\}$. The sets $\{\bar{A}: A \subseteq S\}$ form a basis for the open sets of $S$ as well as a basis for the closed sets of $S$.

Given a discrete semigroup $(S,+)$ the operation extends to $\beta S$ making $(\beta S,+)$ a right topological semigroup (meaning that for each $p \in \beta S$, the function $\rho_{p}: \beta S \rightarrow \beta S$ defined by $\rho_{p}(q)=q+p$ is continuous) with $S$ contained in its topological center (meaning that for each $x \in S$, the function $\lambda_{x}: \beta S \rightarrow \beta S$ defined by $\lambda_{x}(q)=x+q$ is continuous). Given $p, q \in \beta S$ and $A \subseteq S$, we have that $A \in p+q$ if and only if $\{x \in S:-x+A \in q\} \in p$, where $-x+A=\{y \in S: x+y \in A\}$. 


\section{Infinite Matrices}

The definition of universally image partition regularity has a natural generalization for the matrices of order $\omega \times \omega$. We mention here that when we talk of an infinite matrix we shall assume that each row of it contains only finitely many nonzero elements. In the previous section we have seen that if a matrix with entries from $\omega$ is image partition regular over $\mathbb{N}$ then it is universally image partition regular. In this section we see that there are a lots of variety in the infinite case. First we observe that the finite sums matrix

$$
A=\left(\begin{array}{ccccc}
1 & 0 & 0 & 0 & \ldots \\
0 & 1 & 0 & 0 & \ldots \\
1 & 1 & 0 & 0 & \ldots \\
0 & 0 & 1 & 0 & \ldots \\
0 & 1 & 1 & 0 & \ldots \\
1 & 1 & 1 & 0 & \ldots \\
\vdots & \vdots & \vdots & \vdots & \ddots
\end{array}\right)
$$

(whose rows are all vectors with entries from $\{0,1\}$ with only finitely many 1 's and not all 0's) is universally image partition regular. In fact let $(S, \mathcal{A})$ be a pair with $T=\bigcap_{A \in \mathcal{A}}$ clA and $S=\bigcup_{i=1}^{r} C_{r}$. Then by [5, Theorem 4.20] $T$ is a compact right topological semigroup and we choose an idempotent $p \in T$. Hence there exists $i \in\{1,2, \cdots, r\}$ such that $A \cap C_{i} \in p$ for all $A \in \mathcal{A}$. Therefore by [5, Theorem 5.12] there exists a sequence $\left\langle x_{n}\right\rangle_{n=1}^{\infty}$ in $S$ such that $F S\left(\left\langle x_{n}\right\rangle_{n=1}^{\infty}\right) \subseteq A \cap C_{i}$ and therefore we have

$$
A \vec{x} \subseteq A \cap C_{i} .
$$

From [1, Lemma 3.9] it follows that there are infinite image partition regular matrices which are not universally image partition regular.

In fact if we consider the following infinite matrix

$$
M=\left(\begin{array}{ccccc}
1 & 0 & 0 & 0 & \ldots \\
2 & 1 & 0 & 0 & \ldots \\
4 & 0 & 1 & 0 & \ldots \\
8 & 0 & 0 & 1 & \ldots \\
\vdots & \vdots & \vdots & \vdots & \ddots
\end{array}\right)
$$

and $\mathbb{N}$ is finitely colored we can choose a monochromatic sequence $\left\langle y_{n}\right\rangle_{n=0}^{\infty}$ such that $y_{n}>2^{n} y_{0}$ for each $n \in \mathbb{N}$. Let $x_{0}=y_{0}$ and for each $n \in \mathbb{N}$, let $x_{n}=y_{n}-2^{n} y_{0}$. Then $M \vec{x}=\vec{y}$, so that $M$ is $\operatorname{IPR} / \mathbb{N}$. But if we take $\mathcal{A}=\{(0, \epsilon): \epsilon>0\}$ then $M$ is not $\mathrm{IPR} / \mathbb{R}_{\mathcal{A}}^{+}$. In fact if possible let there exists $\vec{x} \in\left(\mathbb{R}^{+}\right)^{\omega}$ such that $\vec{y}=A \vec{x} \in((0,1))^{\omega}$. Then $x_{0}=y_{0}>0$. Pick $k \in \mathbb{N}$ such that $2^{k} x_{0}>1$. Then $y_{k}=2^{k} x_{0}+x_{k}>1$, a contradiction.

Now we shall turn our attention to the Milliken-Taylor matrices with entries from $\omega$ which are one of the main sources of infinite image partition regular matrices over $\mathbb{N}$. In the following theorem we shall prove that these matrices are also universally image partition regular. 
Definition 2.1 Let $m \in \omega, \vec{a}=\left\langle a_{i}\right\rangle_{i=0}^{m}$ be a sequence in $\mathbb{N}$, and $\vec{x}=\left\langle x_{n}\right\rangle_{n=0}^{\infty}$ be a sequence in $S$. Then by Milliken-Taylor system determined by $\vec{a}$ and $\vec{x}$, denoted by $M T(\vec{a}, \vec{x})$ we mean the following set $\left\{\sum_{i=0}^{m} a_{i} \cdot \sum_{t \in F_{i}} x_{t}\right.$ : each $F_{i} \in \mathcal{P}_{f}(\omega)$ and if $i<m$, then $\left.\max F_{i}<\min F_{i+1}\right\}$.

Notice that if $\vec{a}$ has adjacent repeated entries and $\vec{c}$ is obtained from $\vec{a}$ by deleting such repetitions, then for any infinite sequence $\vec{x}$, one has $M T(\vec{a}, \vec{x}) \subseteq M T(\vec{c}, \vec{x})$, so it suffices to consider sequences $\vec{c}$ without adjacent repeated entries.

Definition 2.2 Let $\vec{a}$ be a finite or infinite sequence in $\omega$ with only finitely many nonzero entries. Then $c(\vec{a})$ is the sequence obtained from $\vec{a}$ by deleting all zeroes and then deleting all adjacent repeated entries. The sequence $c(\vec{a})$ is the compressed form of $\vec{a}$. If $\vec{a}=c(\vec{a})$, then $\vec{a}$ is a compressed sequence.

For example, if $\vec{a}=\langle 0,1,0,0,1,2,0,0,2,2,0,0, \ldots\rangle$, then $c(\vec{a})=\langle 1,2\rangle$.

Definition 2.3 Let $\vec{a}$ be a compressed sequence in $\mathbb{N}$. A Milliken-Taylor matrix determined by $\vec{a}$ is an $\omega \times \omega$ matrix $A$ such that the rows of $A$ are all possible rows with finitely many nonzero entries and compressed form equal to $\vec{a}$.

Notice that if $A$ is a Milliken-Taylor matrix whose rows all have compressed form $\vec{a}$ and $\vec{x}$ is an infinite sequence in $S$, then the set of entries of $A \vec{x}$ is precisely $M T(\vec{a}, \vec{x})$.

Definition 2.4 If $(S,+)$ is a discrete semigroup, $p \in \beta S$ and $n \in \mathbb{N}$, then $n \cdot p$ will denote the ultrafilter determined by the set $\{n A: A \in p\}$ where $n A=\{n x: x \in A\}$.

Lemma 2.5 Let $(S, \mathcal{A})$ be a pair, $T=\bigcap_{A \in \mathcal{A}}$ clA and $p=p+p \in T$. Then for any $a \in \mathbb{N}$ we have $a \cdot p \in T$.

Proof. Take $A \in \mathcal{A}$. Then using (1) and (4) of Definition 1.3 and and applying induction we can find $B \in \mathcal{A}$ such that $a B \subseteq A$. Now $B \in p$ so that $a B \in a \cdot p$. Hence $A \in a \cdot p$, and therefore $a \cdot p \in T$.

Theorem 2.6 Let $(S, \mathcal{A})$ be a pair and $\vec{a}=\left\langle a_{i}\right\rangle_{i=0}^{m}$ be a compressed sequence in $\mathbb{N}$, and let $A$ be a Milliken-Taylor matrix determined by $\vec{a}$. Then $A$ is $I P R / S_{\mathcal{A}}$. That is, whenever $r \in \mathbb{N}, S=\bigcup_{i=1}^{r} C_{i}$, and $A \in \mathcal{A}$, there exist $i \in\{1,2, \ldots, r\}$ and a sequence $\left\langle x_{n}\right\rangle_{n=0}^{\infty}$ such that $M T(\vec{a}, \vec{x}) \subseteq C_{i} \cap A$.

Proof. Let $T=\bigcap_{A \in \mathcal{A}} c l A$. Then by [5, Theorem 4.20] $T$ is a compact right topological semigroup so that we can choose an idempotent $p \in T$. Let $q=a_{0} \cdot p+a_{1} \cdot p+\cdots+a_{m} \cdot p$. Then by the above lemma $q \in T$. So it suffices to show that whenever $Q \in q$, there is a sequence $\left\langle x_{n}\right\rangle_{n=0}^{\infty}$ in $S$ such that $M T(\vec{a}, \vec{x}) \subseteq Q$.

Let $Q \in q$ be given. Assume first that $m=0$. Then $\left(a_{0}\right)^{-1} Q \in p$ so there is a sequence $\left\langle x_{n}\right\rangle_{n=0}^{\infty}$ such that $F S\left(\left\langle x_{n}\right\rangle_{n=0}^{\infty}\right) \subseteq\left(a_{0}\right)^{-1} Q$. Then $M T(\vec{a}, \vec{x}) \subseteq Q$. 
Now assume that $m>0$. Then

$$
\left\{y \in S:-y+Q \in a_{1} \cdot p+a_{2} \cdot p+\ldots+a_{m} \cdot p\right\} \in a_{0} \cdot p
$$

so that

$$
P=\left\{x \in S:-\left(a_{0} \cdot x\right)+Q \in a_{1} \cdot p+a_{2} \cdot p+\ldots+a_{m} \cdot p\right\} \in p .
$$

Given $n \in\{1,2, \ldots, m-1\}$ and $x_{0}, x_{1}, \ldots, x_{n-1}$, let $P\left(x_{0}, x_{1}, \ldots, x_{n-1}\right)=\{y \in S$ : $\left.-\left(a_{0} \cdot x_{0}+\ldots+a_{n-1} \cdot x_{n-1}+a_{n} \cdot y\right)+Q \in a_{n+1} \cdot p+\ldots+a_{m} \cdot p\right\}$. If $x_{0} \in P$ and for each $i \in\{1,2, \ldots, n-1\}, x_{i} \in P\left(x_{0}, x_{1}, \ldots, x_{i-1}\right)$, then $P\left(x_{0}, x_{1}, \ldots, x_{n-1}\right) \in p$.

Now given $x_{0}, x_{1}, \ldots, x_{m-1}$, let us set $P\left(x_{0}, x_{1}, \ldots, x_{m-1}\right)=\left\{y \in S: a_{0} \cdot x_{0}+a_{1}\right.$. $\left.x_{1}+\ldots+a_{m-1} \cdot x_{m-1}+a_{m} \cdot y \in Q\right\}$. If $x_{0} \in P$ and for each $i \in\{1,2, \ldots, m-1\}$, $x_{i} \in P\left(x_{0}, x_{1}, \ldots, x_{i-1}\right)$, then $P\left(x_{0}, x_{1}, \ldots, x_{m-1}\right) \in p$.

Given any $B \in p$, let $B^{\star}=\{x \in B:-x+B \in p\}$. Then $B^{\star} \in p$ and by [[5], Lemma 4.14] for each $x \in B^{\star},-x+B^{\star} \in p$.

Choose $x_{0} \in P^{\star}$. Let $n \in \omega$ and assume that we have chosen $x_{0}, x_{1}, \ldots, x_{n}$ such that (1) if $\emptyset \neq F \subseteq\{0,1, \ldots, n\}$, then $\sum_{t \in F} x_{t} \in P^{\star}$, and

(2) if $k \in\{1,2, \ldots, \min \{m, n\}\}, F_{0}, F_{1}, \ldots, F_{k} \in \mathcal{P}_{f}(\{0,1, \ldots, n\})$, and for each $j \in$ $\{0,1, \ldots, k-1\}, \max F_{j}<\min F_{j+1}$, then

$\sum_{t \in F_{k}} x_{t} \in P\left(\sum_{t \in F_{0}} x_{t}, \sum_{t \in F_{1}} x_{t} \ldots, \sum_{t \in F_{k-1}} x_{t}\right)^{\star}$.

Both the hypothesis hold at $n=0,(2)$ vacuously.

For $r \in\{0,1, \ldots, n\}$, let

$$
E_{r}=\left\{\sum_{t \in F} x_{t}: \emptyset \neq F \subseteq\{r, r+1, \ldots, n\}\right\} .
$$

For $k \in\{0,1, \ldots, m-1\}$ and $r \in\{0,1, \ldots, n\}$, let

$$
\begin{aligned}
W_{k, r}=\{ & \left(\sum_{t \in F_{0}} x_{t}, \ldots, \sum_{t \in F_{k}} x_{t}\right): F_{0}, F_{1}, \ldots, F_{k} \in \mathcal{P}_{f}(\{0,1, \ldots, r\}) \\
& \text { and for each } \left.i \in\{0,1, \ldots, k-1\}, \max F_{i}<\min F_{i+1}\right\}
\end{aligned}
$$

Note that $W_{k, r} \neq \emptyset$ if and only if $k \leq r$.

If $y \in E_{0}$, then $y \in P^{\star}$, so $-y+P^{\star} \in p$ and $P(y) \in p$. If $k \in\{1,2, \ldots, m-$ $1\}$ and $\left(y_{0}, y_{1}, \ldots, y_{k}\right) \in W_{k, m}$, then we have $y_{k} \in P\left(y_{0}, y_{1}, \ldots, y_{k-1}\right)$. Which implies that $P\left(y_{0}, y_{1}, \ldots, y_{k}\right) \in p$ and thus $P\left(y_{0}, y_{1}, \ldots, y_{k}\right)^{\star} \in p$. If $r \in\{0,1, \ldots, n-1\}, k \in$ $\{0,1, \ldots, \min \{m-1, r\}\},\left(y_{0}, y_{1}, \ldots, y_{k}\right) \in W_{k, r}$, and $z \in E_{r+1}$, then $z \in P\left(y_{0}, y_{1}, \ldots, y_{k}\right)^{\star}$ so $-z+P\left(y_{0}, y_{1}, \ldots, y_{k}\right)^{\star} \in p$.

If $n=0$, let $x_{1} \in P^{*} \cap\left(-x_{0}+P^{\star}\right) \cap P\left(x_{0}\right)^{\star}$ then the hypotheses are satisfied.

Now assume that $n \geq 1$ and pick

$$
\begin{aligned}
x_{n+1} \in & P^{\star} \cap \bigcap_{y \in E_{0}}\left(-y+P^{\star}\right) \\
& \cap \bigcap_{k=0}^{\min \{m-1, n\}} \bigcap_{\left(y_{0}, \ldots, y_{k}\right) \in W_{k, m}} P\left(y_{0}, \ldots, y_{k}\right)^{\star} \\
& \cap \bigcap_{r=0}^{n-1} \bigcap_{k=0}^{\min \{m-1, r\}} \bigcap_{\left(y_{0}, \ldots, y_{k}\right) \in W_{k, r}} \bigcap_{z \in E_{r+1}}\left(-z+P\left(y_{0}, \ldots, y_{k}\right)^{\star}\right) .
\end{aligned}
$$


For hypothesis (1) assume that $\emptyset \neq F \subseteq\{0,1, \ldots, n+1\}$ and $n+1 \in F$. If $F=\{n+1\}$ we have directly that $x_{n+1} \in P^{\star}$, so assume that $\{n+1\} \subsetneq F$ and let $G=F \backslash\{n+1\}$. Let $y=\sum_{t \in G} x_{t}$. Then $y \in E_{0}$ so $x_{n+1} \in-y+P^{\star}$ and so $\sum_{t \in F} x_{t} \in P^{\star}$.

To this end we verify the hypothesis (2). For this let $k \in\{1,2, \ldots, \min \{m, n+1\}\}$ and assume that $F_{0}, F_{1}, \ldots, F_{k} \in \mathcal{P}_{f}(\{0,1, \ldots, n+1\})$ and for each $j \in\{0,1, \ldots, k-1\}$, $\max F_{j}<\min F_{j+1}$. We can assume that $n+1 \in F_{k}$. For $l \in\{0,1, \ldots, k-1\}$ let $y_{l}=\sum_{t \in F_{l}} x_{t}$. Then $k-1 \leq \min \{m-1, n\}$ and $\left(y_{0}, y_{1}, \ldots, y_{k-1}\right) \in W_{k-1, m}$. If $F_{k}=$ $\{n+1\}$, then $\sum_{t \in F_{k}} x_{t}=x_{n+1} \in P\left(y_{0}, y_{1}, \ldots, y_{k-1}\right)^{\star}$. So assume that $\{n+1\} \subsetneq F_{k}$ and let $F_{k}^{\prime}=F_{k} \backslash\{n+1\}$. Let $r=\max F_{k-1}$. Then $r<\min F_{k}^{\prime}$ so $r \leq n-1, k-1 \leq$ $\min \{m-1, r\}$, and $\left(y_{0}, y_{1}, \ldots, y_{k-1}\right) \in W_{k-1, r}$. Let $z=\sum_{t \in F_{k}^{\prime}} x_{t}$. Then $z \in E_{r+1}$ so $x_{n+1} \in-z+P\left(y_{0}, y_{1}, \ldots, y_{k-1}\right)^{\star}$ so $\sum_{t \in F_{k}} x_{t} \in P\left(\sum_{t \in F_{0}} x_{t}, \sum_{t \in F_{1}} x_{t} \ldots, \sum_{t \in F_{k-1}} x_{t}\right)^{\star}$.

Acknowledgements. The authors are very grateful to the referee for his/her helpful comments which made a serious improvement of the paper.

\section{References}

[1] D. De and N. Hindman Image partition regularity near zero, To appear in Discrete Mathematics

[2] D. De, N. Hindman, and D. Strauss, Sets Central with Respect to Certain Subsemigroups of $\beta S_{d}$, To appear in Topology Proceedings.

[3] N. Hindman and I. Leader, The semigroup of ultrafilters near 0, Semigroup Forum 59 (1999), 33-55.

[4] N. Hindman, I. Leader, and D. Strauss, Infinite partition regular matrices - solutions in central sets, Trans. Amer. Math. Soc. 355 (2003), 1213-1235.

[5] N. Hindman and D. Strauss, Algebra in the Stone-Čech compactification: theory and applications, de Gruyter, Berlin, 1998.

[6] K. Milliken, Ramsey's Theorem with sums or unions, J. Comb. Theory (Series A) 18 (1975), 276-290.

[7] R. Rado, Studien zur Kombinatorik, Math. Zeit. 36 (1933), 242-280.

[8] R. Rado, Note on combinatorial analysis, Proc. London Math. Soc. 48 (1943), 122160.

[9] I. Schur, Über die Kongruenz $x^{m}+y^{m}=z^{m}(\bmod p)$, Jahresbericht der Deutschen Math.-Verein. 25 (1916), 114-117.

[10] A. Taylor, A canonical partition relation for finite subsets of $\omega$, J. Comb. Theory (Series A) 21 (1976), 137-146.

[11] B. van der Waerden, Beweis einer Baudetschen Vermutung, Nieuw Arch. Wiskunde 19 (1927), 212-216. 